\title{
Evaluación del error debido a la evaporación en el método gravimétrico de calibración de micro pipetas
}

\author{
Sica, A. ${ }^{(1)}$, Constantino, P. ${ }^{(1)}$, Heijo, G. ${ }^{(2)}$, Fabretti, J. A. ${ }^{(2)}$, Santo, C. ${ }^{(1)}$ \\ Contacto: asica@latu.org.uy \\ (1) Departamento de Metrología, Laboratorio Tecnológico del Uruguay (LATU) - (2) Aphos SRL. \\ Recibido: 30/7/2009 - Aprobado: 8/12/2009
}

\begin{abstract}
$\underline{\text { Resumen }}$
La calibración de micro pipetas por el método gravimétrico consiste en la carga con agua destilada del instrumento y la posterior descarga en un recipiente abierto. Uno de los errores más frecuentes en este método es el provocado por la evaporación. El objetivo del presente trabajo es cuantificar dicho error y evaluar modificaciones al método gravimétrico convencional que permitan disminuirlo.

Para estudiar la magnitud de este efecto se estima el valor del volumen descargado antes de la evaporación, extrapolando la masa a tiempo cero a partir del gráfico de masa en función del tiempo. Se compara este valor con el obtenido de la lectura directa de la masa luego de la estabilización de la balanza, a un minuto de la descarga.

Se estudia una forma de disminuir la evaporación realizando la descarga en un recipiente con aceite vegetal, ya que por poseer una densidad menor al agua ésta queda ocluida en él. Esta variación del método se compara cuantitativamente con el método gravimétrico convencional con el fin de evaluar la incidencia de esta modificación.

Se concluye que la variación propuesta de descargar en un recipiente con aceite ofrece ventajas operativas, arrojando resultados de calibración comparables.

Palabras clave: gravimetría, evaporación, descarga, aceite, ventajas.
\end{abstract}

\begin{abstract}
$\underline{\text { Abstract }}$
The calibration of piston pipettes by the gravimetric method consists on filling the instrument with distilled water and a subsequent deliver into an open vessel, located on the balance where the weighing is performed. One of the most frequent errors in calibrations by this method is caused by the phenomenon of evaporation. The purpose of this study is to quantify that error and to evaluate modifications to the conventional gravimetric method in order to decrease it.

To study the magnitude of this effect, we estimate the value of the volume delivered before the evaporation process, plotting the mass vs time and extrapolating the value of mass at zero time. This value is compared with the value obtained from direct reading after the stabilization of the balance, that is one minute after dispensing.

We study a way to reduce evaporation performing delivery in a vessel with vegetable oil, since by having a lower density than water the delivery is occluded in, minimizing evaporation. This quantitative variation of this method is compared with the conventional gravimetric method to evaluate the impact of this change in evaporation effect.

We conclude that the alternative procedure of delivering in a vessel with oil provides operational benefits, yielding comparable calibration results.

Keywords: gravimetry, evaporation, discharge, oil, advantages.
\end{abstract}

\section{Introducción}

El método más sencillo y que requiere de menor equipamiento para calibrar micro pipetas es el de gravimetría. Dicho método consiste en descargar con la micro pipeta un líquido de densidad conocida (generalmente agua destilada) en un recipiente cuya masa es medida antes y después de la descarga. Conociendo la masa del líquido y su densidad puede calcularse el volumen descargado.

Los factores que tienen mayor influencia en la incertidumbre de calibración por este método pueden ser el tipo de construcción del instrumento (pistón-cilindro defectuoso, desgaste, etcétera), el propio método (diferencias en la presión de descarga, variaciones en la temperatura y otras condiciones del ensayo) y errores sistemáticos como la evaporación de la descarga de agua en el recipiente de pesaje o calibración de la balanza, entre otros.

El objetivo de este estudio es cuantificar la influencia de la evaporación en los resultados de calibración. Para minimizar la incidencia de la evaporación pueden utilizarse dispositivos disponibles en el mercado que mantienen una atmósfera saturada de humedad en el recipiente de pesada o saturar el ambiente interior de la balanza donde se realiza la descarga.

Para cuantificar los efectos de la evaporación en cualquier condición es posible realizar la descarga en condiciones estándar de la temperatura y humedad, y registrar la lectura de la balanza a distintos tiempos. Con estos valores se realiza la regresión lineal y se obtiene el valor de masa correspondiente a tiempo cero, es decir, en el momento exacto que se realiza la descarga, cuando el efecto por evaporación puede suponerse nulo. Este método permite cuantificar, y por lo tanto corregir, los errores por evaporación, pero incrementa significativamente el tiempo de ensayo y agrega otras fuentes de incertidumbre, como lo es la deriva de la balanza, la medida de tiempo, etcétera.

La alternativa que se propone es utilizar un medio de descarga que disminuya la evaporación de modo que no sea necesario efectuar la corrección por el método de extrapolación. El medio seleccionado es aceite vegetal, el cual por su menor densidad que el agua ocluye la gota de descarga y disminuye así la evaporación. 


\section{Materiales y Métodos}

\section{- Equipos empleados}

Pipeta de volumen variable de $20 \mu 1$ a $200 \mu 1$. Se elige una pipeta de volumen variable. Marca Nichiryo, modelo Nichipet EX, número de serie H7 1004991, división 0,2 $\mu 1$.

Comparador de masa de capacidad máxima 100g. Marca Sartorius, modelo C 100, serie 70501031 y división $0,001 \mathrm{mg}$.

Termómetro de división $0,1{ }^{\circ} \mathrm{C}$ con termocupla. Marca Newport, modelo True RMS, número de serie 6000334 .

Cronómetro marca Casio.

Termohigrómetro y barómetro. Marca testo, modelo 454, número de serie 05604540 y divisiones $0,1^{\circ} \mathrm{C}, 0,1 \% \mathrm{HR}$ y $1 \mathrm{hPa}$.

Recipientes de vidrio. Para la descarga de la pipeta.

Varilla de vidrio.

\section{- $\quad$ Reactivos}

Líquido de calibración. Agua destilada.

Aceite vegetal.

\section{- Procedimiento experimental}

Se evaluarán los resultados obtenidos si se aplican los dos procedimientos descriptos con la variante sugerida (agregado de aceite).

\section{Procedimiento de calibración que compensa}

\section{el efecto de evaporación}

a. Se coloca el tip en la punta de la micro pipeta y se realizan tres enjuagues con agua destilada.

b. Se miden las condiciones ambientales de temperatura, humedad y presión atmosférica, y la temperatura inicial del agua destilada. c. Se coloca el recipiente de pesada en la balanza y se aplica la tara. d. Se pipetea con agua destilada y se vierte en el recipiente de pesada. En ese momento se inicia el conteo del cronómetro. e. Se anotan las lecturas a 30, 60, 90, 120 y 150 segundos. f. Por último se registra la temperatura final del agua destilada.

\section{Modificación realizada}

Se coloca en recipiente de pesada un volumen de aceite muy superio al volumen de agua utilizado en todo el proceso de calibración y se sigue el procedimiento detallado anteriormente. En este caso debe tenerse la precaución de que toda el agua descargada quede cubierta por el aceite. Con este fin se utiliza la varilla, de manera de sumergir la gota con movimientos suaves.

\section{Procedimiento simplificado (no compensa el efecto de evaporación)}

Este procedimiento es igual al anterior pero en el paso e) sólo se registra la lectura a los 60 segundos de la descarga.

También se realiza la modificación propuesta (agregado de aceite) para este procedimiento.

En todos los casos se toman medidas con la micro pipeta en volúmenes nominales de $50 \mu 1$ y $200 \mu 1$, registradas por dos operadores y con un número de cinco repeticiones cada uno.

\section{- Procesamiento de datos}

Modelo matemático para procedimiento de calibración que compensa el efecto de evaporación

Este procedimiento considera la realización de medidas de masa descargada a distintos tiempos. Se realiza una regresión lineal de los resultados de masa obtenidos en cada ensayo a 30, 60, 90, 120 y 150 segundos, de manera de obtener la pendiente y la ordenada en el origen en cada caso. La pendiente representa la evaporación media (variación media de la masa de agua descargada en el recipiente en función del tiempo), mientras que la ordenada en el origen representa la masa descargada a tiempo cero según el modelo matemático aplicado.

Para el tratamiento de datos se calcularon los promedios de todas las medidas realizadas por los dos operadores que participaron en cada ensayo.

El volumen descargado por la micro pipeta a $20^{\circ} \mathrm{C}$ se calcula según la siguiente ecuación (ec. 1):

$$
V_{20}=\left(M_{d}\right) \cdot\left(\frac{1}{\rho_{W}-\rho_{A}}\right) \cdot\left(1-\frac{\rho_{A}}{\rho_{B}}\right) \cdot(1-\gamma \cdot(t-20))
$$

Donde:

$M_{d}$ es promedio de las masas a tiempo cero (extrapoladas a partir de las regresiones lineales realizadas) $(\mathrm{g})$.

$\rho_{A}$ es la densidad del aire $(\mathrm{g} / \mathrm{ml})$.

$\rho_{B}$ es la densidad de las pesas utilizadas en la calibración de la balanza.

$\rho_{W}$ es la densidad del agua a $\mathrm{t}^{\circ} \mathrm{C}(\mathrm{g} / \mathrm{ml})$.

$t$ es la temperatura del agua de calibración.

$\gamma$ es el coeficiente de dilatación térmica del material del instrumento (en el caso de micro pipetas corresponderá al coeficiente de expansión térmica del material del sistema de pistón del instrumento, en este caso se usa el correspondiente al polipropileno de $\left.\gamma=1,0 \mathrm{E}-05 \quad 1 /{ }^{\circ} \mathrm{C}\right)$.

\section{Modelo matemático para procedimiento simplificado}

En este modelo no se corrige el efecto por evaporación, calculándo se el volumen de la micro pipeta a partir de la masa descargada media a un minuto de realizada la descarga.

Para ello se utiliza la ecuación (ec. 1), pero usando el promedio de las lecturas de la balanza a un minuto de la descarga. Todos los valores promediados están en las mismas condiciones de temperatura y ambientales, teniendo en cuenta las incertidumbres en las variables de influencia. Por esta razón es válido promediar las masas descargadas en lugar de los volúmenes.

\section{Estimación de incertidumbre}

La incertidumbre correspondiente a ambos procedimientos de calibración fue calculada como la suma cuadrática de las componentes tipo A y tipo B (según ISO/IEC 98-3).

\section{Evaluación del error por evaporación}

Como forma de evaluar si el error sistemático debido a la evaporación es significativo, se propone normalizarlo, es decir, calcular el error normalizado como indica la siguiente ecuación (ec. 2):

$$
E n=\frac{E}{\sqrt{\left(U_{\text {ref }}^{2} \cdot U_{\text {resultado })}^{2}\right.}}
$$

$E n=$ Error normalizado

$E=$ Resultado - Valor de referencia (error debido a la evaporación). $U_{\text {ref }}=$ Incertidumbre expandida del valor de referencia.

$U_{\text {resultado }}^{\text {ref }}=$ Incertidumbre expandida del resultado informado. 
Como valor de referencia se utilizará en todos los casos el valor de calibración obtenido a partir del método de descarga en recipiente seco, considerando la evaporación. Un error normalizado superior a 1 implica que el error en la medición es significativo.

\section{Resultados y Discusión}

\section{Resultados del procedimiento de calibración que compensa el efecto de evaporación}

Las Tablas 1 y 2 expresan los resultados para los volúmenes nominales de $50 \mu 1$ y $200 \mu 1$, respectivamente, utilizando las dos variantes del método.

\begin{tabular}{|c|c|c|}
\hline & Aqua & Accite \\
\hline Temperatura media $\left({ }^{\circ} \mathrm{C}\right)$ & 20,17 & 20,76 \\
\hline Wolumen a $20^{\circ} \mathrm{C}(\mathrm{il})$ & 50,12 & 50,14 \\
\hline Error $(\mu i)$ & 0,12 & 0,14 \\
\hline Incertidumbre expandida ( $\mu$ ) & 0,29 & 0,19 \\
\hline Evaporación media (mg:s-1) & $-4,9 E-03$ & $-4,4 E-05$ \\
\hline Repetibilidad pendiente (mg $s^{-1}$ ) & $8,2 E-03$ & $7,0 \mathrm{E}-05$ \\
\hline Evaporaciōn en un minuto (ul) & 0.30 & 0.05 \\
\hline
\end{tabular}

Tabla 1. Resultados obtenidos para el volumen de $50 \mu 1$.

\begin{tabular}{|c|c|c|}
\hline & Agua & Aceite \\
\hline Temuperafura media (C) & 20,63 & 20,63 \\
\hline Volumen a $20^{\circ} \mathrm{C}(\mu \mathrm{H})$ & 200.50 & 200,56 \\
\hline Error $(\mu i)$ & 0,50 & 0,56 \\
\hline Incertidumbre expundida (p) & 0,24 & 0,60 \\
\hline Evaporación media (mgs.'s') & $-7,7 \mathrm{E}-03$ & $-1,5 E-04$ \\
\hline Repetibilidad pendiente (mg.s-1) & $1,4 E-03$ & $1,6 E-04$ \\
\hline Evaporaciön en un minuto (ul) & 0,61 & 0.01 \\
\hline
\end{tabular}

Tabla 2. Resultados obtenidos para el volumen de $200 \mu 1$.

En el Gráfico 1 se muestran los promedios de las pendientes correspondientes a las regresiones lineales para las dos variantes del método.

Esta pendiente es una medida de la pérdida de masa de agua por evaporación con respecto al tiempo en ambas variantes. Se observa en el caso de la descarga en aceite que el efecto de la evaporación puede considerarse despreciable frente a los valores correspondientes a la descarga en el recipiente seco. Por otro lado, se observa que utilizando un recipiente seco la pendiente de evaporación es mayor para el mayor volumen nominal. Esto se debe a que estas pendientes no son relativas al volumen. Si se relativizan al volumen, las pendientes de evaporación son menores para mayores volúmenes, ya que la relación área expuesta/volumen es menor en este caso.

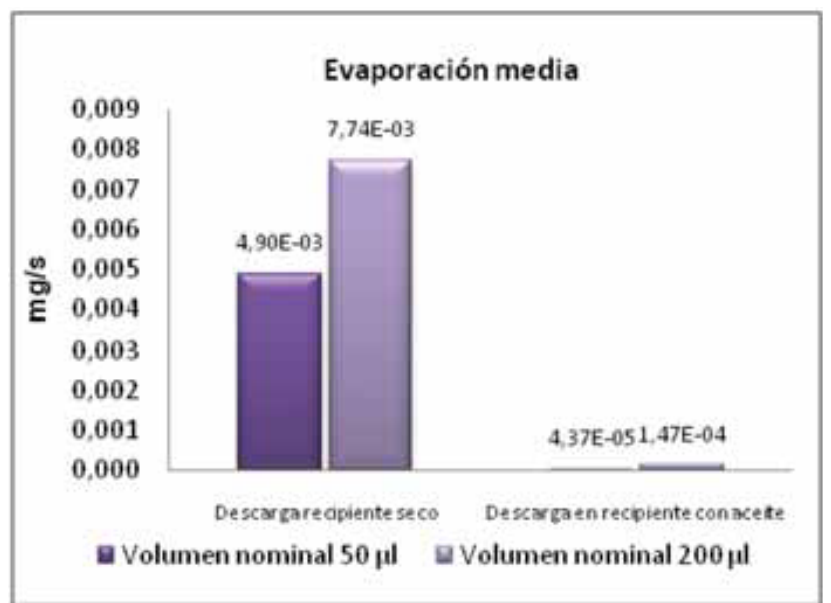

Gráfico 1. Promedios de las pendientes de las regresiones lineales de cada método.
En los Gráficos 2 y 3 se observan los resultados de la calibración por ambas variantes (recipiente seco y con aceite). Estos resultados son comparables dentro de las incertidumbres estimadas.

Se concluye que si se corrige el efecto de evaporación con el modelo matemático propuesto, las dos variaciones conducen a resultados equivalentes, implicando evaporaciones muy diferentes. Esto puede considerarse una validación de la exactitud del método de descarga en recipiente seco con extrapolación a tiempo cero, haciendo posible la utilización de estos resultados como valores de referencia en la evaluación de la exactitud del método de descarga en aceite.

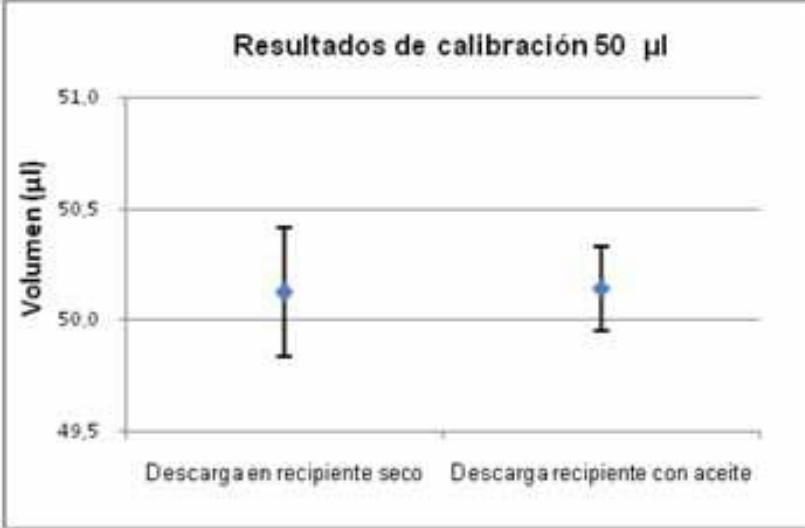

Gráfico 2. Volumen de calibración con su incertidumbre para la pipeta en $50 \mu 1$

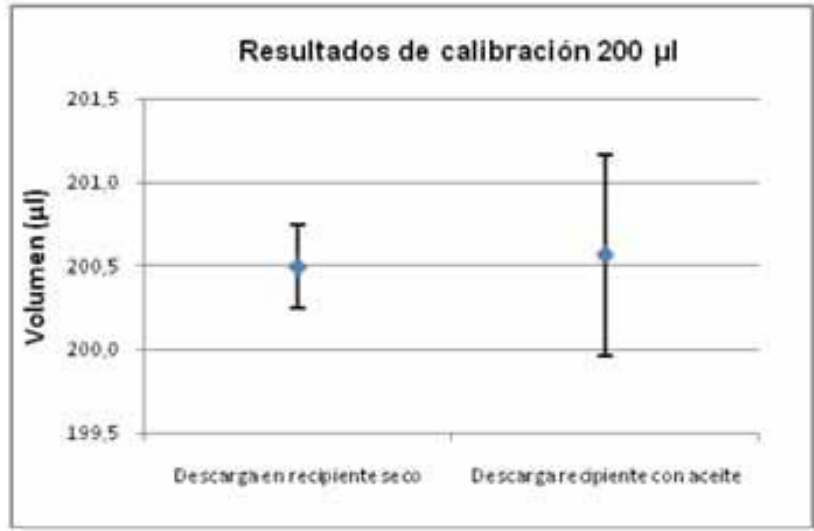

Gráfico 3. Volumen de calibración con su incertidumbre para la pipeta en $200 \mu 1$.

\section{Resultados del procedimiento de calibración simplificado (no compensa el efecto de evaporación)}

Para la cuantificación del volumen de evaporación se tomaron las lecturas a un minuto con las dos variantes propuestas. Esto es debido a que en los ensayos del método de descarga en aceite la lectura de la balanza a 30 segundos no alcanza la estabilidad.

Se observa que el método de descarga en aceite disminuye el volumen de agua evaporado, reduciendo el error sistemático debido al efecto de la evaporación en el proceso de calibración. En el Gráfico 4 se observa el volumen evaporado a un minuto de la descarga en ambos métodos. 


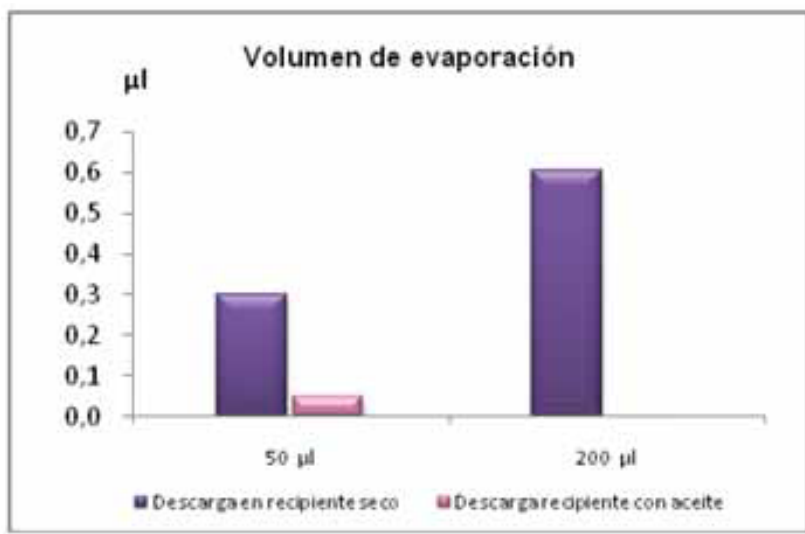

Gráfico 4. Promedios de las pendientes de las regresiones lineales de cada método.

En los Gráficos 5 y 6 se comparan los resultados de calibración obtenidos en todos los procedimientos, tomando como referencia el correspondiente a la descarga en recipiente seco y compensando la pérdida por evaporación.

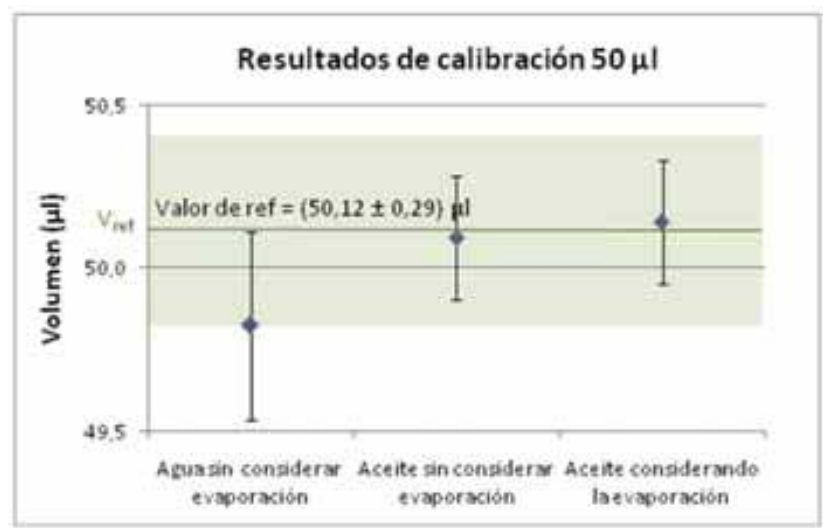

Gráfico 5. Resultados de calibración para volumen nominal de $50 \mu 1$.

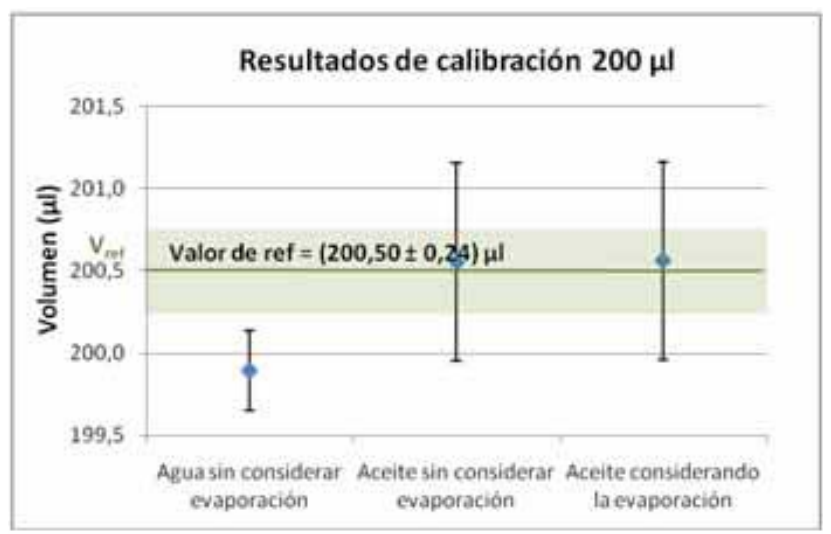

Gráfico 6. Resultados de calibración para volumen nominal de $200 \mu$ l.

En los Gráficos 5 y 6 puede observarse que los valores que se obtienen realizando la calibración descargando en el recipiente seco sin compensar la pérdida por evaporación, tienen un sesgo significativo, mientras que los resultados descargando en aceite son comparables al valor de referencia en ambos casos para los dos volúmenes ensayados. Esto se comprueba numéricamente en la Tabla 3, donde se observan los errores normalizados de los ensayos graficados anteriormente. Los valores cercanos y aun superiores a 1 de los resultados al realizar la descarga en agua sin compensar la pérdida de masa por evaporación manifiestan la importancia de considerar este efecto. Por otra parte, al realizar la calibración descargando en aceite los errores normalizados son muy inferiores a 1.

\begin{tabular}{|l|c|c|}
\hline Ensayo & $\mathbf{5 0} \mathbf{\mu l}$ & $\mathbf{2 0 0} \mathrm{\mu l}$ \\
\hline Descarga en agua" & 0,73 & 1,76 \\
\hline Descarga en aceite" & 0,06 & 0,10 \\
\hline Descarga en aceite & 0,08 & 0,09 \\
\hline
\end{tabular}

${ }^{a}$ Compensando la pérdida por evaporación (por extrapolación de la masa a tiempo cero) ${ }^{\mathrm{b}} \mathrm{Sin}$ compensar la pérdida por evaporación (tomando como resultado el correspondiente a la lectura a 1 minuto de la descarga)

Tabla 3. Resultados de errores normalizados para ambos volúmenes nominales.

\section{Conclusiones}

Los resultados obtenidos demuestran que considerar la evaporación (método de calibración simplificado) permite evitar errores sistemáticos significativos.

La extrapolación a tiempo cero es una forma efectiva de obtener resultados de calibración confiables, ya que estima el valor de masa en el momento de la descarga.

El método de descarga en aceite como forma alternativa de eliminar el efecto de evaporación ofrece ventajas operativas a la calibración, dado que permite realizar el procedimiento sin tomar medidas a lo largo del tiempo, con resultados comparables.

\section{Reconocimientos}

Se agradece a las siguientes personas por sus aportes para la realización de este trabajo:

Al Quím. Farmac. Andrés Venturini, por proporcionarnos reactivos necesarios para este trabajo.

A la Dra. Mary Lopretti, por proporcionarnos la micro pipeta utilizada en este trabajo.

A la Ing. Quím. Joselaine Cáceres, por ceder el uso de las instalaciones del laboratorio de masa para realizar las medidas.

\section{Referencias}

\section{- INTERNATIONAL STANDARD ORGANIZATION}

(Switzerland). ISO 4787: Laboratory glassware, volumetric glassware. Methods for use and testing of capacity. Geneva: ISO, 1984.

- INTERNATIONAL STANDARD ORGANIZATION

(Switzerland). ISO 8655-2: Piston operated volumetric apparatus.

Part 2: Piston pipettes. Geneva: ISO, 2002.

- INTERNATIONAL STANDARD ORGANIZATION

(Switzerland). ISO/IEC 98-3: Guide to the Expression of

Uncertainty in Measurement. Geneva: ISO, 2008. 